\title{
Human exhaled air can efficiently support in vitro maturation of porcine oocytes and subsequent early embryonic development
}

\author{
Zubing Cao, Di Gao, Tengteng Xu, Xu Tong, Yiqing Wang, Yunsheng Li, Fugui Fang, Jianping Ding, \\ Xiaorong Zhang, Yunhai Zhang ${ }^{1}$
}

Anhui Provincial Laboratory of Local Livestock and Poultry, Genetical Resource Conservation and Breeding, College of Animal Science and Technology, Anhui Agricultural University, Hefei 230036, China.

\begin{abstract}
Air phase is an indispensable environmental factor affecting oocyte maturation and early embryo development. Human exhaled air was previously proved to be a reliable and inexpensive atmosphere that sustains normal early development of mouse and bovine embryos. However, whether human exhaled air can support in vitro maturation (IVM) of porcine oocytes is not yet known. To evaluate the feasibility of maturing oocytes in human exhaled air, we examined oocyte morphology, BMP15 expression, nuclear and cytoplasmic maturation. We found that cumulus expansion status, expression levels of BMP15 important for cumulus expansion and the rate of first polar body emission were similar among human exhaled air, 5\% $\mathrm{O}_{2}$ or $20 \% \mathrm{O}_{2}$ in air after IVM of $44 \mathrm{~h}$. Furthermore, the percentage of metaphase II (MII) oocytes showing normal cortical and sub-membranous localization of cortical granules and diffused mitochondrial distribution patterns is comparable among groups. The cleavage, blastocyst rate and total cell number were not apparently different for parthenogenetic activated and somatic cloned embryos derived from MII oocytes matured in three air phases, suggesting oocytes matured in human exhaled air obtain normal developmental competence. Taken together, human exhaled air can efficiently support in vitro maturation of porcine oocytes and subsequent early embryonic development.
\end{abstract}

Keywords: human exhaled air, pig, oocyte maturation, somatic cell nuclear transfer, early embryo.

\section{Introduction}

The faithful achievement of mammalian oocyte maturation is an essential prerequisite to carry out the robust preimplantation embryo development(Gilchrist and Thompson, 2007; Coticchio et al. 2015). Oocyte maturation mainly consists of two key biological events involving a set of complex nuclear and cytoplasmic changes that determine oocyte quality(Sun and Nagai, 2003; Krisher, 2004; Wang and Sun, 2007). At present, the external culture conditions of oocyte maturation in vitro are not yet optimal compared with naturally physiological environment in the reproductive tract (Fischer and Bavister, 1993; Roberts et al., 2002). This suboptimal culture environment may induce many detrimental roles in oocytes in vitro maturation and subsequent early embryonic development. Indeed, previous studies indicated that abnormal meiotic maturation could lead to a series of developmental defects involving meiotic progression arrest, aneuploidy in eggs and embryos, failure of pronucleus formation and even mitotic chaos in early embryonic development (Howe and FitzHarris, 2013; Hu et al., 2015; MacLennan et al., 2015). In addition, incomplete cytoplasmic maturation including abnormal relocalization of cortical granules and mitochondria, and spatio-temporally translational failure of maternal mRNA, could cause dysfunctional epigenetic reprogramming and mitotic events important for fertilization, embryonic genome activation and blastocyst formation (Pocar et al., 2001; Dumollard et al., 2007; Watson 2007; Chen et al., 2013; Huan et al., 2015). To date, accumulating evidence revealed that the developmental competency (also termed oocyte quality) of the resulting matured oocytes mostly depends on external culture environment during oocyte maturation in vitro (Lonergan et al., 2003; Wrenzycki and Stinshoff, 2013). Therefore, a stable and reliable incubation system for oocyte maturation in vitro is very important and deserves plenty of attention. In fact, it is discovered that culture conditions during in vitro maturation of oocyte encompass many facets, for example, compositions of culture medium, temperature, humidity, carbon dioxide and oxygen concentrations(Bavister and Poole, 2005; Iwamoto et al., 2005; Park et al., 2005; Swain, 2010; Wrenzycki and Stinshoff, 2013). The majority of studies focuses on the optimization of composition of culture medium used for oocyte maturation in vitro, however, other environmental factors affecting oocyte maturation, such as culture air phase, also need to be examined.

So far, air phases commonly utilized for oocyte maturation in vitro are mainly separated into two categories involving 5\% $\mathrm{CO}_{2}, 20 \% \mathrm{O}_{2}, 75 \% \mathrm{~N}_{2}$ (high oxygen tension; Bavister, 1995) and 5\% $\mathrm{CO}_{2}, 5 \% \mathrm{O}_{2}$, 95\% $\mathrm{N}_{2}$ (low oxygen tension)(Adam et al., 2004; Kang et al. 2012). There are some disadvantages for in vitro maturation of oocytes using these two air phases. First, expensive standard incubator must be purchased and used to maintain the correct concentration of commercially available mixed gas stored in the cylinder. Second, consumption and cost of mixed air is also extremely expensive. Third, internal culture environment including temperature, humidity and $\mathrm{CO}_{2}$ equilibrium are often perturbed due to frequent openings of the incubator door which could result in 
suboptimal oocyte maturation and subsequent embryo development. Fourth, it is very difficult and dangerous for long-distance transportation of these two incubation apparatuses and is also not convenient to utilize them under field conditions, especially in animal farms. Thus, a simple, cost-effective, stable and reliable incubation system is desired to meet the need of oocyte maturation in the remote labs and animal farms. Two earlier studies demonstrated that mouse and bovine embryos cultured in vitro in the aluminium bag inflated with human exhaled air consisting of $4 \% \mathrm{CO}_{2}, 16-17 \% \mathrm{O}_{2}$, and $79-80 \% \quad \mathrm{~N}_{2}$ were able to develop to blastocyst stage(Tarkowski and Wroblewska, 1967; Vajta, 1997). However, whether this incubation system using human exhaled air could be employed to mature in vitro oocytes remains to be known. In this study, we used porcine oocytes as a model to investigate whether human exhaled air could support the normal nuclear and cytoplasmic maturation of porcine oocytes as well as subsequent early embryonic development.

\section{Materials and Methods}

\section{Ethics statement}

All animal experiments were conducted according to the Institutional Animal Care and Use Committee guidelines at Anhui Agricultural University.

\section{Chemicals and antibodies}

All chemicals were purchased from Sigma (St Louis, MO) unless otherwise stated. MitoTracker Red CMXRos was purchased from Invitrogen (Cat. No: M7510).

\section{Preparation of human exhaled air incubation system}

Airtight aluminium bag was sterilized by $75 \%$ alcohol in PBS and then these bags were naturally dried at room temperature. Four-well plates including porcine cumulus-oocyte complexes (COCs) were placed into the sterilized aluminium bag and then experimenter sealed the aluminium bag by sealing machine. A plastic tube equipped with 18-gauge syringe needle was used to connect bag with experimenters. Subsequently, healthy researchers started to exhale air into bag. Experimenter again sealed the bag to ensure the complete airtightness when it was inflated with appropriate exhaled air. Finally, the bag was placed into a constant temperature incubator without the inflation of commercial mixture gas and incubated for $44 \mathrm{~h}$ to allow the oocytes maturation. At the same time, the aluminium bag system was also used to culture COCs of the $5 \% \mathrm{CO}_{2} / 5 \% \mathrm{O}_{2}$ and $5 \% \mathrm{CO}_{2} / 20 \% \mathrm{O}_{2}$ groups.

\section{Oocyte in vitro maturation (IVM)}

This experiment was performed as described previously (Cao et al., 2014). Briefly, ovaries from prepubertal gilts were collected at a local slaughterhouse and transported to the laboratory at $28^{\circ} \mathrm{C}-35^{\circ} \mathrm{C}$ in physiological saline solution containing penicillin
(0.2 IU/mL) and streptomycin sulfate $(0.2 \mathrm{IU} / \mathrm{mL})$. The ovaries were washed in saline and the ovarian follicles from 3 to $6 \mathrm{~mm}$ in diameter were aspirated using a sterile $10 \mathrm{~mL}$ syringe with an 18-gauge needle attached. The aspirated follicular fluid was slowly injected into a preincubated $15 \mathrm{~mL}$ centrifuge tube to sediment the cumulus-oocyte complexes (COCs). The COCs with more than three layers of cumulus cells and homogeneous ooplasm were selected under a stereomicroscope. In vitro maturation (IVM) medium (TCM-199 supplemented with 15\% FBS, $10 \mathrm{ng} / \mathrm{mL}$ EGF, $10 \%$ porcine follicular fluid, $10 \mathrm{IU} / \mathrm{mL}$ of eCG, $5 \mathrm{IU} / \mathrm{mL}$ of hCG, $0.8 \mathrm{mM}$ L-glutamine and $0.05 \mathrm{mg} / \mathrm{mL}$ gentamicin) was pre-equilibrated overnight at $38.5^{\circ} \mathrm{C}$, human exhaled air, 5\% $\mathrm{CO}_{2} / 5 \% \mathrm{O}_{2}$ and $5 \% \mathrm{CO}_{2} / 20 \%$ $\mathrm{O}_{2}$ in air, respectively. Subsequently, 50 of the COCs were washed and transferred in 4-well plate containing $400 \mu \mathrm{L}$ IVM medium and the bags filled with three different gases were simultaneously put in a constant temperature incubator with $38.5^{\circ} \mathrm{C}$ for $42-44 \mathrm{~h}$. The COCs were then treated with DPBS without $\mathrm{Ca}^{2+}$ and $\mathrm{Mg}^{2+}$ (Gibco, Grand Isle, NY) containing $1 \mathrm{mg} / \mathrm{mL}$ hyaluronidase to remove the surrounding cumulus cells. Finally, oocytes with clear perivitelline spaces, intact cell membranes, and extruded the first polar body (pb1) were selected for subsequent experiment.

\section{Parthenogenetic activation}

Oocytes with first polar body emission were activated parthenogenetically by two pulses of direct current $(1.56 \mathrm{kV} / \mathrm{cm}$ for $80 \mathrm{~ms})$ in activation medium (280 mM mannitol, $0.1 \mathrm{mM} \mathrm{CaCl}_{2}$, and $0.1 \mathrm{mM} \mathrm{MgCl}_{2}$ ). Subsequently, embryos were washed in PZM-3 three times, followed by $4 \mathrm{~h}$ of incubation in the chemically assisted activation medium (PZM-3 supplemented with $10 \mu \mathrm{g} / \mathrm{mL}$ cycloheximide and $10 \mu \mathrm{g} / \mathrm{mL}$ cytochalasin B) covered by paraffin oil. Embryos were then washed three times with PZM-3 medium and cultured in fresh PZM-3 medium at $38.5^{\circ} \mathrm{C}, 5 \% \mathrm{CO}_{2}$ and $95 \%$ air with saturated humidity.

\section{Preparation of donor cells}

Landrace fetus in 35 days old was recovered and rinsed three times with PBS. The left tissues after removing head, intestine, liver, heart and limbs were cut into small pieces and incubated in fetal bovine serum (FBS). The tissue blocks were evenly smeared in a dish and cultured upside down at $37^{\circ} \mathrm{C}, 5 \% \quad \mathrm{CO}_{2}$ and saturated humidity. After $8 \mathrm{~h}$ of incubation, fibroblast cells were transferred into the standard cell culture medium (FBS supplemented with 85\% DMEM, $0.1 \mathrm{mM}$ NEAA, and $0.05 \mathrm{mM}$ L-glutamine). Fetal fibroblast cells were dissociated and passaged until 90\% confluence. Fetal fibroblast cells with 4th-8th generations were used as donor cells for nuclear transfer.

\section{Somatic cell nuclear transfer (SCNT)}

SCNT was performed as described previously (Cao et al., 2015). Briefly, denuded MII oocytes were enucleated in manipulation medium (TCM199 
supplemented with $2 \%$ FBS and $7.5 \mu \mathrm{g} / \mathrm{mL}$ cytochalasin B) through removing the first polar body and a small amount of the surrounding cytoplasm containing spindle using a 15-20 mm beveled glass pipette. Donor cells were injected into the enucleated oocyte to generate reconstructed couplets. Electric pulse was applied to the couplets in fusion medium, then immediately placed in PZM-3 medium. After $30 \mathrm{~min}$ of incubation, fused embryos were further incubated for $4 \mathrm{~h}$ in chemically assisted activation medium at $38.5^{\circ} \mathrm{C}$ and $5 \% \mathrm{CO}_{2}$ with saturated humidity. Finally, embryos were washed three times using fresh PZM-3 medium and cultured in fourwell plates containing PZM-3 medium at $38.5^{\circ} \mathrm{C}$ and $5 \% \mathrm{CO}_{2}$ in humidified air.

\section{Evaluation of cortical granules distribution}

The zona pellucida of MII oocytes was removed in $0.5 \%$ pronase solution, followed by washing in DPBS containing $0.3 \%$ BSA three times. Oocytes were fixed in $4 \%$ paraformaldehyde in DPBS for $30 \mathrm{~min}$, followed by washing in DPBS supplemented with $0.3 \%$ BSA and $10 \mathrm{mM}$ glycine. Oocytes were subsequently permeabilized in $0.1 \%$ Triton $\mathrm{X}-100$ for $5 \mathrm{~min}$ at room temperature, followed by washing in DPBS containing $0.3 \%$ BSA two times. Oocytes were then labeled with $100 \mu \mathrm{g} / \mathrm{mL}$ FITC-conjugated peanut agglutinin (Sigma, L7381) in DPBS for 30 min in a dark chamber. Finally, the oocytes were washed three times in DPBS containing $0.3 \%$ BSA and $0.01 \%$ Triton X-100. DNA was labeled after staining with $10 \mu \mathrm{g} / \mathrm{mL}$ PI in DPBS for $10 \mathrm{~min}$, followed by washing three times in DPBS, and then mounted on glass slides. Oocytes were imaged under an epifluorescence microscope (Olympus, IX71, Japan).
Oocytes omitting the primary antibody were used as negative controls to examine the specificity of the reaction.

\section{Assessment of mitochondria distribution}

$1 \mathrm{mM}$ stock solution of MitoTracker Red CMXRos was prepared in DMSO and stored at $-20^{\circ} \mathrm{C}$. Denuded MII oocytes were incubated for $30 \mathrm{~min}$ in IVM medium supplemented with $0.5 \mu \mathrm{M} / \mathrm{L}$ MitoTracker Red CMXRos at $38.5^{\circ} \mathrm{C}$ and $5 \% \mathrm{CO}_{2}$ in humidified air. Oocytes were then washed three times in DPBS containing $0.3 \%$ BSA. Subsequently, oocytes were fixed in in $4 \%$ paraformaldehyde in DPBS for $30 \mathrm{~min}$, followed by washing in DPBS supplemented with $0.3 \%$ BSA and $0.01 \%$ Triton X-100. Finally, the chromosomes of oocytes were labeled with $10 \mu \mathrm{g} / \mathrm{mL}$ Hoechst33342 in DPBS for $10 \mathrm{~min}$, followed by washing three times in DPBS, and then mounted on glass slides. Oocytes were imaged under an epifluorescence microscope (Olympus, IX71, Japan).

\section{Quantitative real-time PCR ( $q R T-P C R)$}

Total RNA was isolated from denuded oocytes matured at $18 \mathrm{~h}$ using the RNeasy Micro Kit (Qiagen, cat.No.74004). cDNA synthesis was performed using a QuantiTect Reverse Transcription Kit (Qiagen, cat.No.205311). Real-time qPCR analysis was conducted using StepOne Plus (Applied Biosystems). Reactions were performed technically in triplicate and were repeated biologically three times. The housekeeping gene EF1A1 was used as the endogenous control. The primer sequences used were listed in Table 1.

Table 1. Sequence information on porcine-specific primers for quantitative real-time polymerase chain reaction.

\begin{tabular}{|c|c|c|c|}
\hline Gene & Primer sequence $\left(5^{\prime}-3^{\prime}\right)$ & Product size (bp) & GenBank accession no. \\
\hline \multirow{2}{*}{ BMP15 } & F: CGCCATCAACTTCACCTAGCT & \multirow{2}{*}{120} & \multirow{2}{*}{ NM_001005155.1 } \\
\hline & R: CAGCAGGGAAGGCTTTAAGG & & \\
\hline \multirow{2}{*}{ EF $1 \alpha 1$} & F: AATGCGGTGGGATCGACAAA & \multirow{2}{*}{120} & \multirow{2}{*}{ NM_001097418.1 } \\
\hline & R: CACGCTCACGTTCAGCCTTT & & \\
\hline
\end{tabular}

Abbreviations: F, forward; R, reverse.

\section{Statistical analysis}

All experiments were repeated at least three times. All data were expressed as mean \pm standard error of mean (mean \pm S.E.M) and SPSS (Version 17.0) was used to perform single factor analysis of variance (ANOVA) for the percentage of polar body extrusion, 2-cell, blastocyst, total cell number per blastocyst, BMP15 expression, oocyte with cortical and peripheral sub-membranous distribution of cortical granules and oocyte with mitochondrial distribution in the cytoplasm. $\mathrm{P}<0.05$ was considered statistically significant.

\section{Results}

Human exhaled air maintains normal nuclear maturation of porcine oocytes

To explore the effects of different oxygen tensions on the nuclear maturation in vitro of immature porcine oocytes, we cultured cumulus-oocytes complexes (COCs) for $44 \mathrm{~h}$ under different air phase conditions involving human exhaled air, $5 \% \quad \mathrm{O}_{2}$ or $20 \% \mathrm{O}_{2}$ in air. Two air phases including $5 \% \mathrm{O}_{2}$ and $20 \%$ $\mathrm{O}_{2}$ in air that are commonly used to culture oocytes were set as positive control. Assessment of nuclear maturation rate was indicated by the percentage of first polar body extrusion. At first, the whole procedures of human exhaled air preparation including three important steps were shown in Figure 1A. The peripheral compacted cumulus cells surrounding oocytes cultured in three air phases have significantly expanded and loosed after $44 \mathrm{~h}$ of culture and the expansion extent of the cumulus cells appeared to be comparable among different groups (Fig. 1B). Moreover, there was not significantly different in the rate of first polar body extrusion among three groups (67\% versus $60 \%, 63 \%$; Fig. 1C). Therefore, these results indicate that human exhaled air is sufficient to support the meiotic maturation in vitro of porcine oocytes. 
A Human exhaled air collection
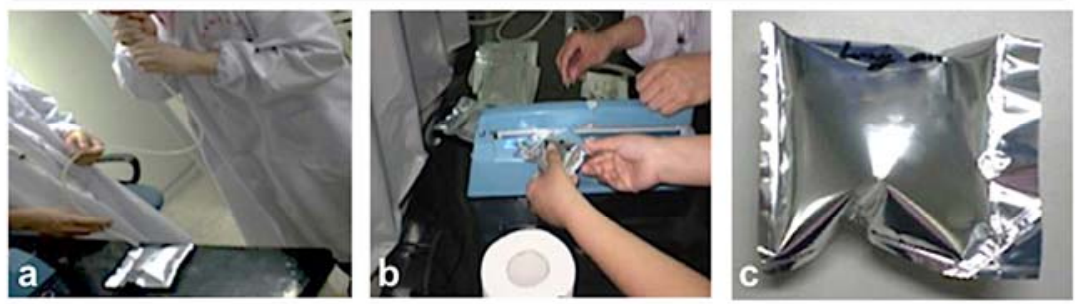

B
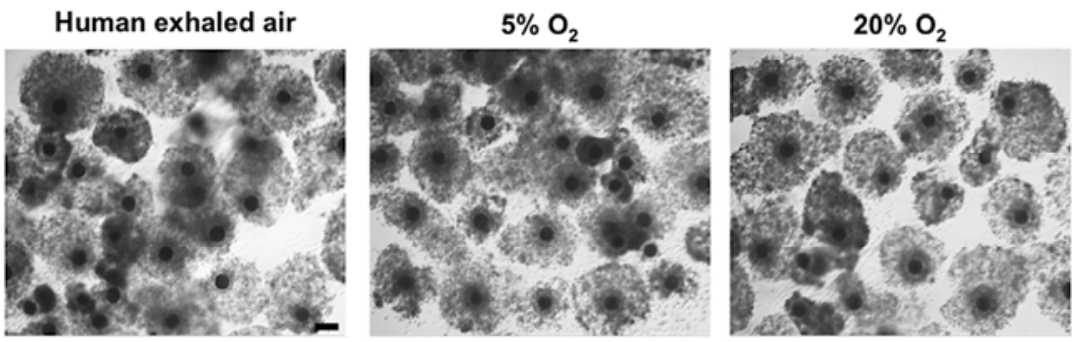

C

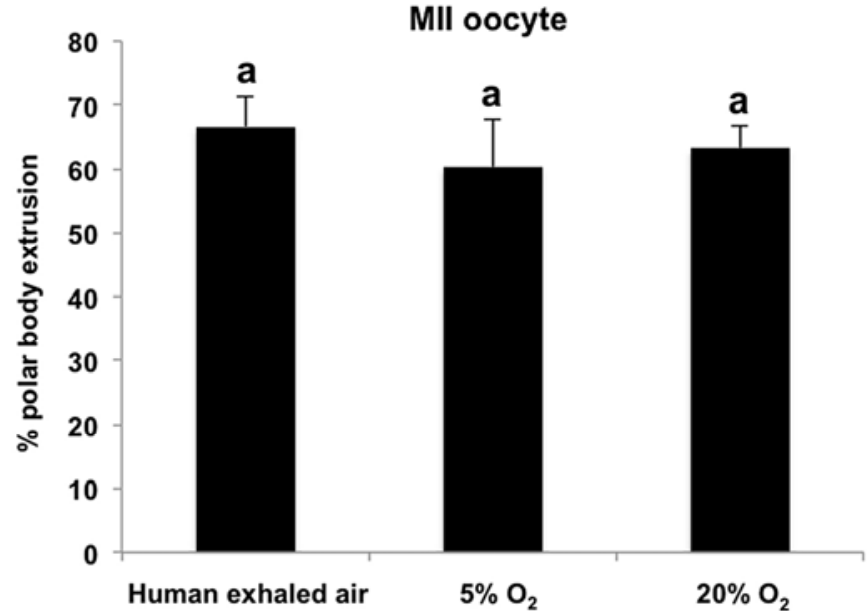

Figure 1. Human exhaled air maintains normal meiotic maturation of porcine oocytes. (A) The procedures of human exhaled air preparation. a. Porcine cumulus-oocyte complexes (COCs) at germinal vesicle (GV) stage were cultured in four-well plates containing in vitro maturation (IVM) medium covered with mineral oil. Four-well plates were transferred into the sterilized airtight aluminium bag and then experimenters exhaled air into the bag. b. Outside edge of bag should be sealed immediately when bag was inflated with appropriate human exhaled air. c. Shown was an aluminium bag inflated with human exhaled air. (B) Representative images of COCs with expanded cumulus cells matured in vitro in different air phases for $44 \mathrm{~h}$. Scale bars: $100 \mu \mathrm{m}$. (C) Quantitative analysis of polar body extrusion (PBE) rate for COCs matured in vitro in different air phases for $44 \mathrm{~h}$. The experiment was conducted four times with $240 \mathrm{GV}$ oocytes per group. All the percentage data are expressed relative to the number of GV oocytes and shown as mean \pm S.E.M. Values with different superscripts across groups indicate significant differences $(\mathrm{P}<0.05)$. MII denotes metaphase stage of meiosis II.

Human exhaled air sustains robust distribution of cortical granules and mitochondria during porcine oocyte maturation

The distribution patterns of cortical granules and mitochondria are usually used to assess the status of cytoplasmic maturation in mammalian oocytes after meiotic maturation (Sha et al., 2010). Furthermore, previous studies showed that migration of cortical granules (CGs) to the cortical area is a common hallmark in oocyte meiotic maturation (Wessel et al., 2002). Based on this information, we matured COCs in three air phases to metaphase II (MII) stage indicated by the first polar body extrusion and further analyzed the proportion of MII oocyte displaying cortical area and peripheral sub-membranous distribution. We found that CGs in the majority of MII oocytes matured in three air phases exhibited the distribution pattern of cortical and peripheral sub-membranous area (Fig.2 A). Besides, the percentage of MII oocytes displaying cortical and peripheral sub-membranous localization of CGs is similar among three groups (Fig. 2B). Since mitochondrial distribution in cortical area and inner region of oocyte cytoplasm has been observed in matured in vitro porcine MII oocytes (Yang et al., 2010), we only here examined mitochondria with the certain distribution pattern in MII oocytes matured under different air phase conditions. Interestingly, we 
observed that mitochondria in most of MII oocytes matured in three air phases evenly diffused throughout the whole cytoplasm and cortical area (Fig. 2C). Moreover, there was no difference in the percentage of MII oocytes showing the cytoplasmic and cortical area distribution of mitochondria among three groups (Fig. 2D). Altogether, these results indicate that human exhaled air can maintain the normal distribution patterns of CGs and mitochondria to ensure the robust cytoplasmic maturation of porcine oocytes.

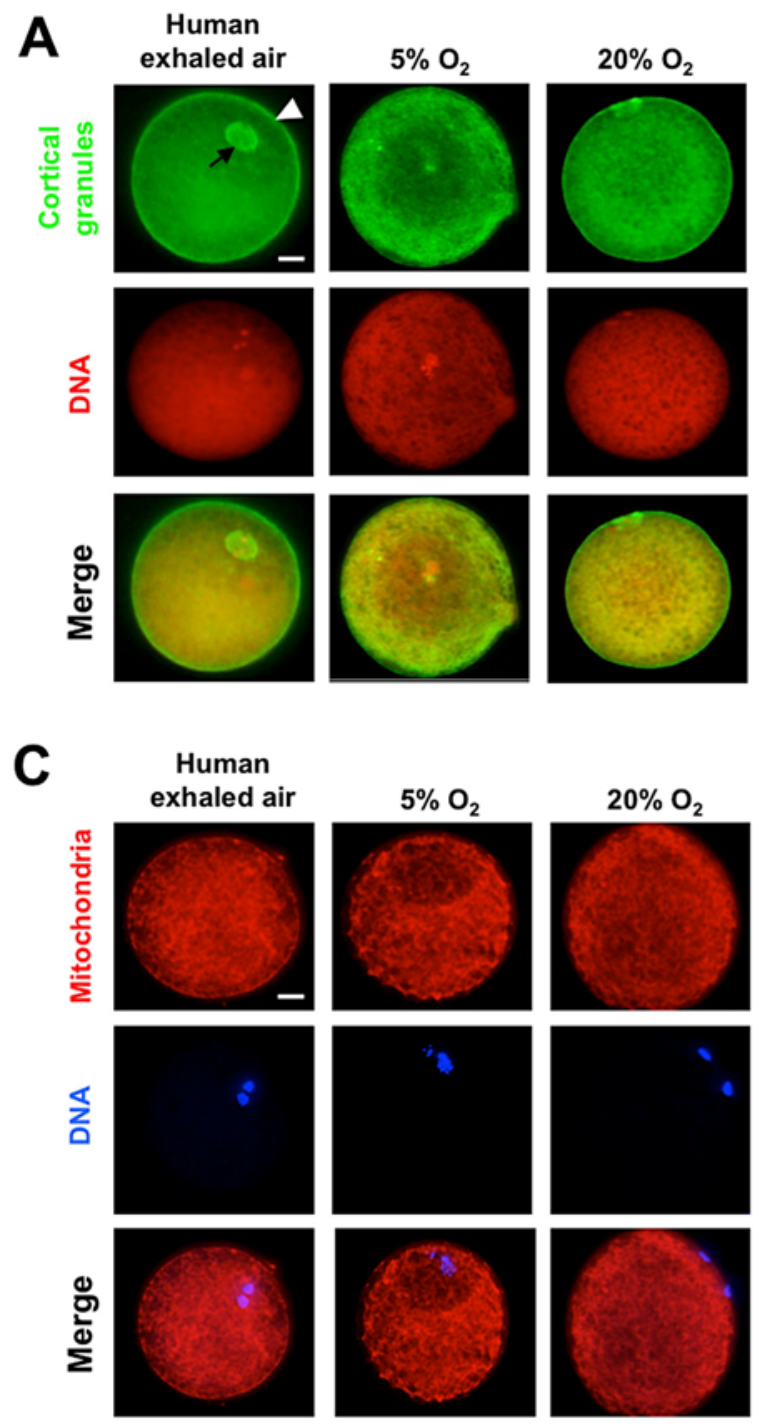

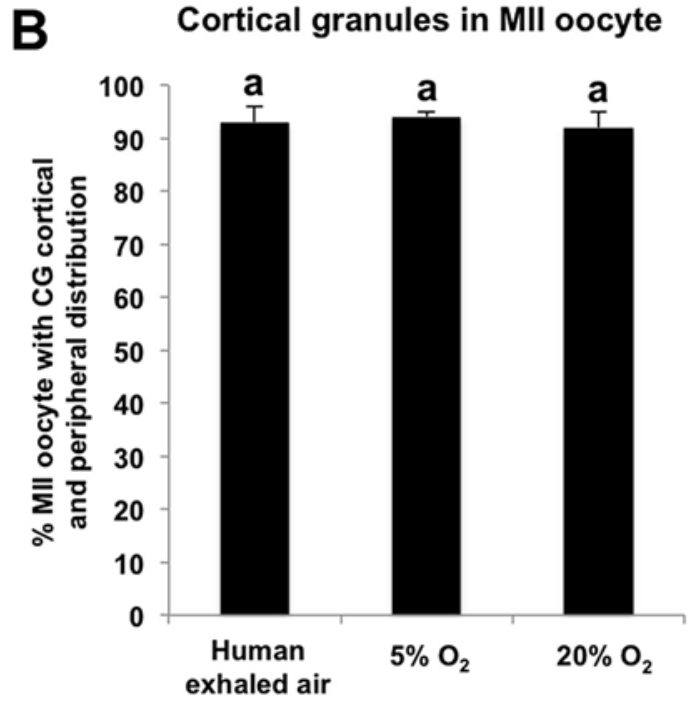

D

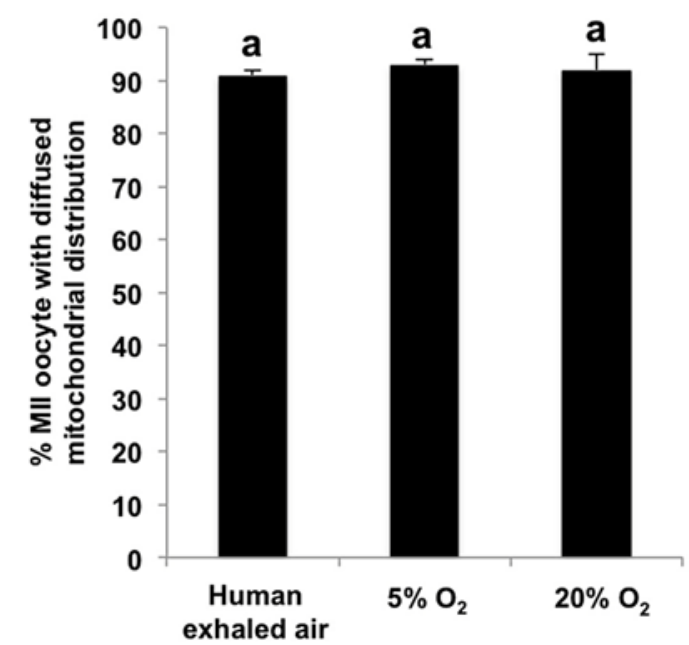

Figure 2. Human exhaled air sustains robust distribution of cortical granules and mitochondria during porcine oocyte maturation. (A) Immunofluorescence analysis of cortical granules (CG) with cortical area and peripheral submembranous distribution in denuded metaphase-II (MII) oocytes cultured in different air phases. Cortical granules and DNA in denuded MII oocytes were labeled with FITC-conjugated peanut agglutinin (green) and propidium iodide (red). Bottom panels showed the merged images (yellow) between cortical granules signals and DNA staining. Shown are representative Z-stacks obtained by epifluorescence microscopy from one experiment. Arrow indicates the first polar body, arrowhead denotes sub-membranous region. Scale bars: $20 \mu \mathrm{m}$. (B) Quantification of the percentage of MII oocytes with GC displaying cortical area and peripheral sub-membranous distribution in A. Data are shown as mean \pm S.E.M. The experiment was repeated three times with 60 oocytes (human exhaled air), 64 oocytes $\left(5 \% \mathrm{O}_{2}\right)$, 48 oocytes $\left(20 \% \mathrm{O}_{2}\right)$, respectively. Values with different superscripts across groups indicate significant differences $(\mathrm{P}<0.05)$. (C) Immunofluorescence analysis of active mitochondria with diffused distribution in denuded metaphase-II (MII) oocytes cultured in different air phases. Mitochondria and DNA were labeled with MitoTracker Red CMXRos (red) and Hoechst33342 (blue). Bottom panels showed the merged images between cortical granules signals and DNA staining. Shown are Z-stacks obtained by epifluorescence microscopy from a representative experiment. Scale bars: $20 \mu \mathrm{m}$. (D) Quantification of the percentage of MII oocytes exhibiting diffused mitochondrial distribution in C. Data are shown as mean \pm S.E.M. The experiment was performed three times with 44 oocytes (human exhaled air), 56 oocytes $\left(5 \% \mathrm{O}_{2}\right), 52$ oocytes $\left(20 \% \mathrm{O}_{2}\right)$, respectively. Values with different superscripts across groups indicate significant differences $(\mathrm{P}<0.05)$. 
Human exhaled air maintains correct expression of BMP15 essential for porcine oocyte maturation

Oocyte-secreted factor BMP15 is a critical regulator for cumulus expansion during oocyte maturation (Buccione et al., 1990) and the maximum expression abundance of BMP15 gene was observed in porcine oocytes matured in vitro for $18 \mathrm{~h}$ (Li et al., 2008). To examine whether oocytes matured in vitro under different air phase conditions have normal expression levels of BMP15, we employed qPCR to detect the expression abundance of BMP15 in porcine oocytes matured in vitro for $18 \mathrm{~h}$ (Fig. 3A). qPCR analysis showed that BMP15 expression level was not statistically different among three groups (Fig. 3B). Hence, our results indicate that human exhaled air does not alter the expression abundance of BMP15 required for porcine oocyte maturation.

A

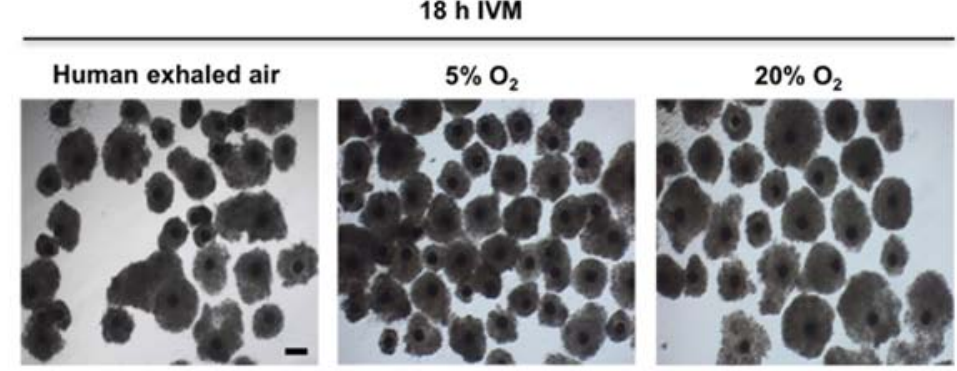

Figure 3. Human exhaled air maintains correct expression of BMP15 essential for porcine oocyte maturation. (A) Representative images of COCs matured in vitro in different air phases for $18 \mathrm{~h}$. Scale bars: $100 \mu \mathrm{m}$. (B) Realtime qPCR analysis of BMP15 transcripts in denuded oocytes derived from COCs of A. Expression levels were normalized against endogenous housekeeping gene EF1 $\alpha 1$ and human exhaled air group was set to 1 . A total of three biological replicates were performed. Data are shown as mean \pm S.E.M. Values with different superscripts across groups indicate significant differences $(\mathrm{P}<0.05)$. IVM denotes in vitro maturation.

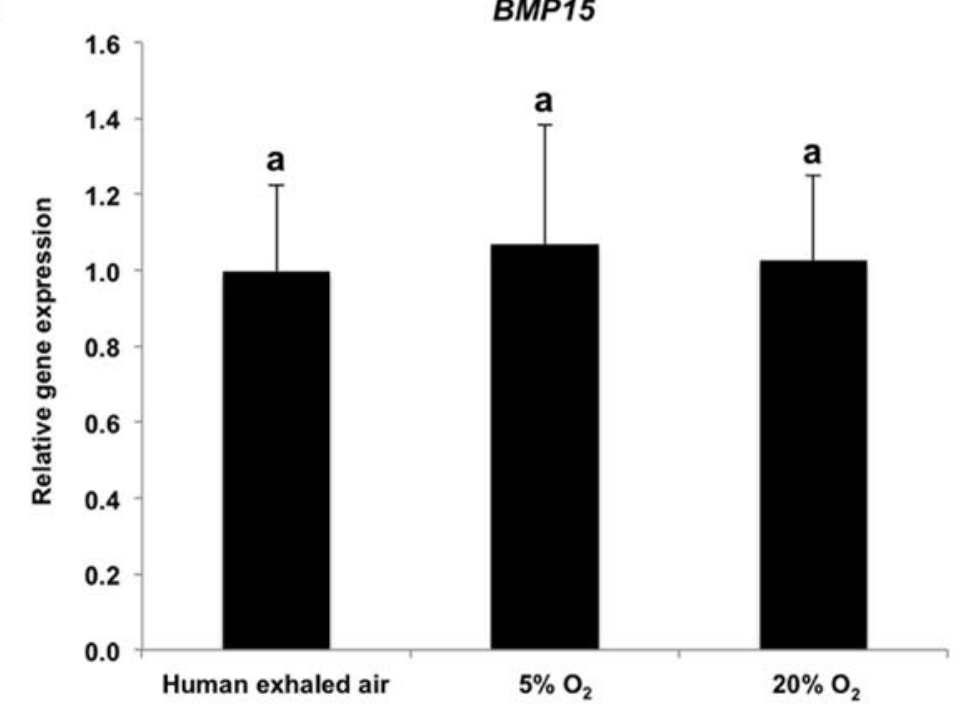

Oocytes matured in human exhaled air support the early development of parthenogenetic activated embryos

Given that oocytes matured in human exhaled air have normal nuclear and cytoplasmic maturation, we want to explore whether the resulting oocytes cultured in human exhaled air support the early development of parthenogenetic activated (PA) embryos. MII oocytes matured in vitro under different air phase conditions were parthenogenetically activated and cultured for 7 days in a humidified incubator at $38.5^{\circ} \mathrm{C}$ and $5 \% \quad \mathrm{CO}_{2}$. There was no significant difference in the cleavage rate, blastocyst rate and total cell number per blastocyst among groups (Fig. 4A, B, C, D). Therefore, these data demonstrate that oocytes matured in human exhaled air are able to support the early development of parthenogenetic

activated embryos.

Oocytes matured in human exhaled air support the early development of somatic cloned embryos

To investigate whether enucleated oocytes matured in human exhaled air can reprogram the terminally differentiated somatic cells to the totipotent state, we examined the early developmental competency of somatic cloned embryos derived from oocytes matured under different air phase conditions. There was no significant difference in the cleavage rate, blastocyst rate and total cell number per blastocyst among groups (Fig. 5A, B, C, D). Therefore, these data suggest that oocytes matured in human exhaled air are able to successfully perform the reprogramming events to ensure the normal early development of somatic cloned embryos. 

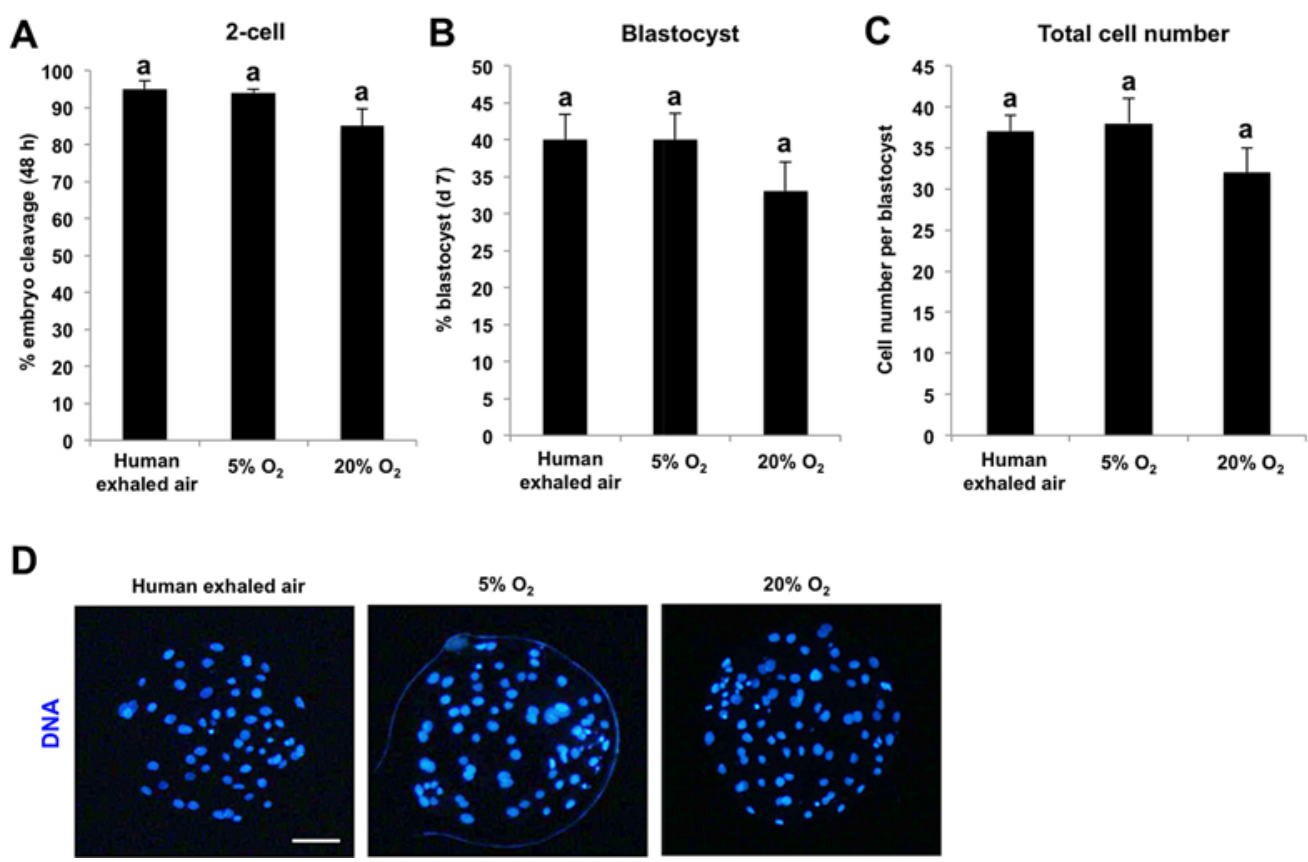

Figure 4. Oocytes matured in human exhaled air support the early development of parthenogenetic activated embryos. Metaphase II (MII) oocytes matured in vitro in different air phases were parthenogenetically activated (PA) by electric pulse. PA embryos were then cultured for 7 days in fresh PZM-3 medium at $38.5 \mathrm{C}$ and $5 \% \mathrm{CO} 2$ in humidified air. The cleavage rate (48 h) (A), blastocyst rate (day 7) (B) and total cell number per blastocyst (C) were statistically analyzed. Data are shown as mean \pm S.E.M. The experiment was repeated four times with 240 embryos per group. Values with different superscripts across groups indicate significant differences $(P<0.05)$. (D) Representative images of PA blastocysts derived from MII oocytes matured in vitro in different air phases. DNA was labeled with Hoechst33342 (blue). Scale bars: $100 \mu \mathrm{m}$.
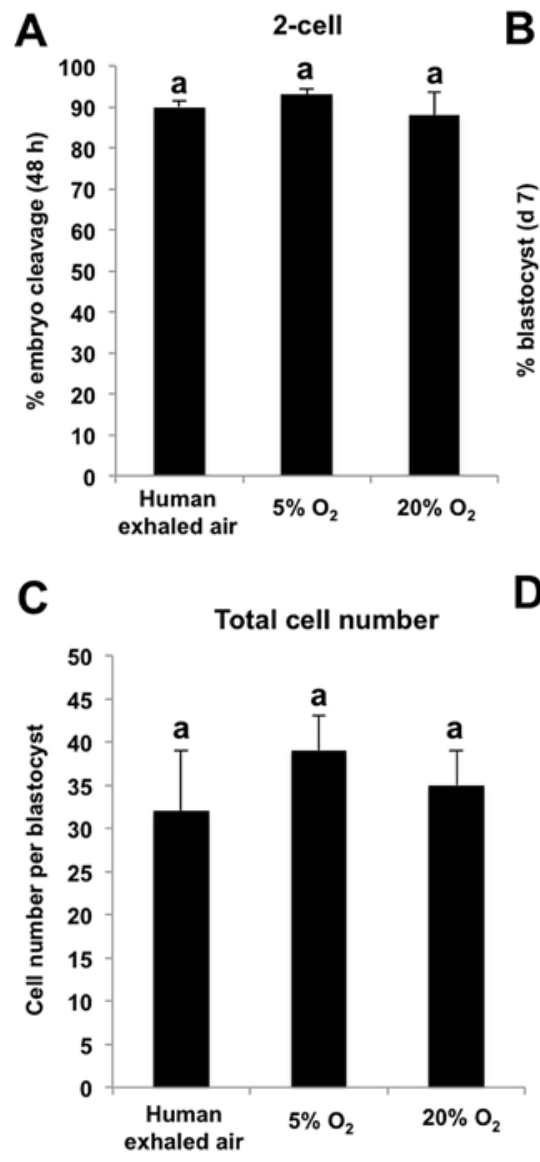

B
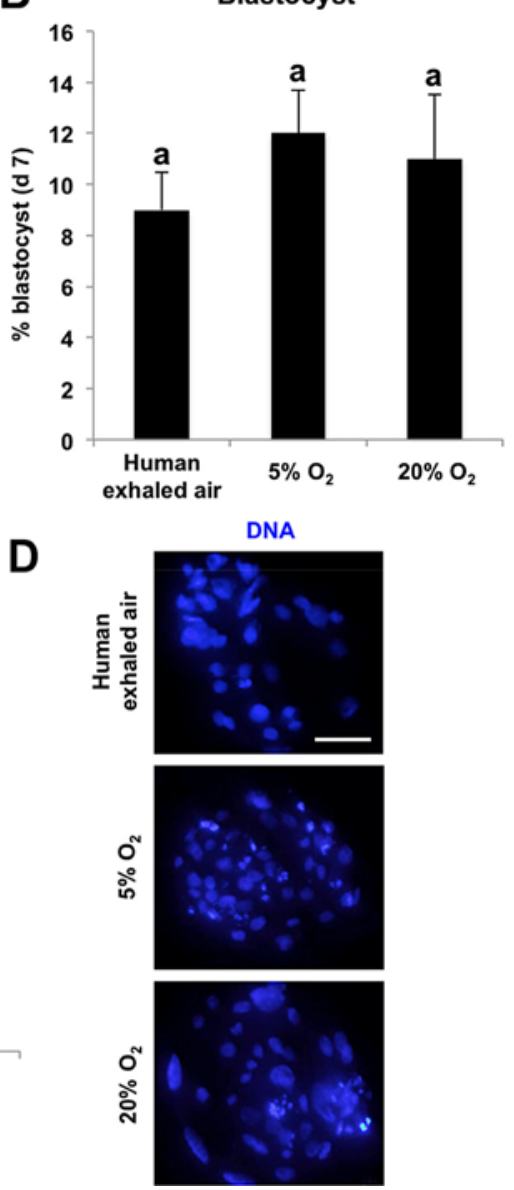

Figure 5. Oocytes matured in human exhaled air support the early development of somatic cloned embryos. Somatic cell nuclear transfer (SCNT) embryos were generated though transplanting donor cells into enucleated MII oocytes matured in vitro in different air phases. SCNT embryos were then cultured in fresh PZM-3 medium at $38.5^{\circ} \mathrm{C}$ and $5 \% \mathrm{CO}_{2}$ in humidified air for 7 days. The cleavage rate (48 h) (A), blastocyst rate (day 7) (B) and total cell number per blastocyst (C) were statistically analyzed. Data are shown as mean \pm S.E.M. The experiment was repeated four times with 120 embryos per group. Values with different superscripts across groups indicate significant differences ( $\mathrm{P}<0.05)$. (D) Representative images of SCNT blastocysts derived from MII oocytes matured in vitro in different air phases. DNA was labeled with Hoechst33342 (blue). Scale bars: $100 \mu \mathrm{m}$. 


\section{Discussion}

In the present study we discovered that human exhaled air can efficiently support the nuclear and cytoplasmic maturation of porcine oocytes from which parthenogenetic activated and somatic cloned embryos derived have comparable developmental efficiency and quality with two traditional air phases (Kang et al., 2012). This incubation system is an inexpensive, portable and reliable approach that is alternative to the traditional large gas-filled incubator and external gas tank. Therefore, it is very suitable to culture oocytes in the remote labs and animal farms, and even acts as a back-up gas supply apparatus to the current labs.

Previous study indicated that oxygen concentration is the main different composition among three air phases in which oxygen level in human exhaled air parallels to $20 \% \mathrm{O}_{2}$ in air, but higher than $5 \% \mathrm{O}_{2}$ in air (Vajta, 1997; Wang and Sun, 2007). Oxygen gas is thought to be an indispensable environmental factor influencing oocyte maturation. Furthermore, low oxygen tension in vitro paralleling to the physiological oxygen level in animal reproductive tract could be benefit to the oocyte maturation (Iwamoto et al., 2005). However, we did not find the analogous phenomenon in our study because the rate of meiotic maturation is similar among human exhaled air, $5 \% \mathrm{O}_{2}$ and $20 \% \mathrm{O}_{2}$ in air. Consistent with our results, low oxygen concentration during in vitro maturation (IVM) has no significant effects on nuclear maturation of porcine oocytes (Park et al., 2005). This discrepancy could be due to the diverse maturation mediums or protocols used in the different labs. Anyway, human exhaled air used in the simple incubation system does not at least exert the visible adverse effects on nuclear maturation of porcine oocytes compared to other two traditional air phases.

To further examine whether human exhaled air affects the cytoplasmic maturation of porcine oocytes, we assessed the distribution patterns of cortical granules (CG) and mitochondria in metaphase II (MII) stage oocytes and expression levels of BMP15 in oocytes matured at $18 \mathrm{~h}$. It is well known that CGs migration and mitochondrial distribution are two clear hallmarks of oocyte cytoplasmic maturation for many species (Wessel et al., 2002). Previous studies showed that CGs migrate to the cortical and sub-membranous area during porcine oocyte maturation(Sha et al., 2010; Yang et al., 2010; Zhang et al., 2010). The disorganization of CGs would reduce the oocyte quality and even impaired subsequent embryo developmental competence (Huan et al., 2015). Our results indicated that most of CGs migrated to cortical and sub-membranous area in MII oocytes matured in three air phases and no significant difference was also observed for the percentage of MII oocytes with cortical localization of CGs. The relocation pattern of CGs in porcine MII oocytes in our study is consistent with the data in other study in which porcine oocytes were incubated in traditional air phase (Sha et al., 2010). Oocytes need to obtain the robust activity and localization of mitochondria to ensure the successful completion of oocyte cytoplasmic
maturation(Van Blerkom and Runner, 1984). Indeed, whether or not correct relocation of mitochondria is tightly related with the developmental competence of oocytes (Brevini et al., 2005; Brevini et al., 2007). It is reported that mitochondria should translocate from periphery area at GV stage to the inner cytoplasmic regions at MII stage during porcine oocyte maturation both in vitro (Sun et al., 2001) and in vivo (Torner et al., 2004). In line with this data, in our study the mitochondria in the majority of MII oocytes matured in three air phases uniformly diffused in the cytoplasm. Therefore, human exhaled air could maintain the correct distribution patterns of CGs and mitochondria to ensure the acquisition of sufficient cytoplasmic maturation.

BMP15 is an oocyte-secreted factor important for oogenesis and folliculogenesis in mice (Yan et al., 2001). In sheep, BMP15 is reported to be critical for ovulation and fertility (McNatty et al., 2005). In pig, dynamic expression of BMP15 during oocyte maturation has been characterized. Specifically, BMP15 not only initiates its expression, but also reaches to the maximum level at $18 \mathrm{~h}$ of IVM culture, which is consistent with the beginning of cumulus expansion ( $\mathrm{Li}$ et al., 2008). Therefore, BMP15 may be important for cumulus cell expansion during porcine oocyte maturation. In our study, there was no significant difference in BMP15 expression levels in oocytes matured in vitro for $18 \mathrm{~h}$ among three air phases. This imply that human exhaled air could maintain normal BMP15 expression compared to other two air phases.

Maternally inherited RNA and proteins during oocyte growth and maturation is considered important for reprogramming of sperm or somatic cells to totipotent embryos (Miyamoto et al., 2011; Zheng et al., 2013; Kong et al., 2014). Especially, cytoplasmic maturation involving appropriate synthesis of maternal RNA is necessary for embryonic genome activation and blastocyst formation (Chen et al., 2013). Thus far, the relatively accurate method to assess cytoplasmic maturation status is tracking the developmental competence of early embryos (Krisher, 2004). Although cytoplasmic maturation of porcine oocytes matured in human exhaled air appeared to be robust, as indicated by the cellular features of CGs and mitochondria distribution, whether these matured oocytes can support the early development of embryos derived from parthenogenetic activation (PA) and somatic cell nuclear transfer (SCNT) need to be confirmed. We found here that the cleavage rate, blastocyst rate and total cell number per blastocyst did not differ among different groups. These results further confirmed that porcine oocytes matured in human exhaled air successfully perform cytoplasmic maturation during IVM, which is sufficient to sustain the early development of PA and SCNT embryos. Further researches are required to classify whether these matured oocytes cultured under human exhaled air condition can produce somatic cloned piglets.

In conclusion, human exhaled air is a simple, cost-effective, portable and reliable atmospheric phase that can efficiently support in vitro maturation of porcine oocytes and subsequent early embryonic development. 


\section{Acknowledgments}

This work was supported by the National Transgenic New Species Breeding Program of China (2014ZX08006-01B), Natural Science Foundation of the Higher Education Institutions of Anhui Province (KJ2016A227), Anhui Provincial Natural Science Foundation (1708085QC55) and Startup Foundation of Anhui Agricultural University for Talented Scholar (YJ2016-10).

\section{Conflicts of Interest}

The authors declare no competing or financial interests in this research.

\section{Author Contributions}

Conceived and designed the experiments: ZBC YHZ. Performed the experiments: ZBC RYH HL BD. Analyzed the data: ZBC YSL YHL. Discussed the results of the experiment: JPD YSL YHL FGF. Contributed reagents/materials/analysis tools: YHZ. Wrote the paper: ZBC XRZ YHZ.

\section{References}

Adam AA, Takahashi Y, Katagiri S, Nagano M. 2004. Effects of oxygen tension in the gas atmosphere during in vitro maturation, in vitro fertilization and in vitro culture on the efficiency of in vitro production of mouse embryos. Jpn J Vet Res, 52:77-84.

Bavister BD. 1995. Culture of preimplantation embryos: facts and artifacts. Hum Reprod Update, 1:91-148.

Bavister BD, Poole KA. 2005. Duration and temperature of culture medium equilibration affect frequency of blastocyst development. Reprod Biomed Online, 10:124-129.

Brevini TA, Vassena R, Francisci C, Gandolfi F. 2005. Role of adenosine triphosphate, active mitochondria, and microtubules in the acquisition of developmental competence of parthenogenetically activated pig oocytes. Biol Reprod, 72:1218-1223.

Brevini TA, Cillo F, Antonini S, Gandolfi F. 2007. Cytoplasmic remodelling and the acquisition of developmental competence in pig oocytes. Anim Reproduction Sci, 98:23-38.

Buccione R, Vanderhyden BC, Caron PJ, Eppig JJ. 1990. FSH-induced expansion of the mouse cumulus oophorus in vitro is dependent upon a specific factor(s) secreted by the oocyte. Dev Biol, 138:16-25.

Cao Z, Li Y, Chen Z, Wang H, Zhang M, Zhou N, Wu R, Ling Y, Fang F, Li N, Zhang Y. 2015. GenomeWide Dynamic Profiling of Histone Methylation during Nuclear Transfer-Mediated Porcine Somatic Cell Reprogramming. PloS one, 10:e0144897.

Cao Z, Zhou N, Zhang Y, Wu R, Li Y, Li N. 2014. Dynamic reprogramming of 5-hydroxymethylcytosine during early porcine embryogenesis. Theriogenology, 81:496-508.

Chen J, Torcia S, Xie F, Lin CJ, Cakmak H, Franciosi F, Horner K, Onodera C, Song JS, Cedars
MI, Ramalho-Santos M, Conti M. 2013. Somatic cells regulate maternal mRNA translation and developmental competence of mouse oocytes. Nat Cell Biol, 15:14151423.

Coticchio G, Dal Canto M, Mignini Renzini M, Guglielmo MC, Brambillasca F, Turchi D, Novara PV, Fadini R. 2015. Oocyte maturation: gametesomatic cells interactions, meiotic resumption, cytoskeletal dynamics and cytoplasmic reorganization. Hum Reprod Update, 21:427-454.

Dumollard R, Duchen M, Carroll J. 2007. The role of mitochondrial function in the oocyte and embryo. Curr Top Dev Biol, 77:21-49.

Fischer B, Bavister BD. 1993. Oxygen tension in the oviduct and uterus of rhesus monkeys, hamsters and rabbits. J Reprod Fertil, 99:673-679.

Gilchrist RB, Thompson JG. 2007. Oocyte maturation: emerging concepts and technologies to improve developmental potential in vitro. Theriogenology, 67:615.

Howe K, FitzHarris G. 2013. Recent insights into spindle function in mammalian oocytes and early embryos. Biol Reprod, 89:1-9.

Hu MW, Wang ZB, Teng Y, Jiang ZZ, Ma XS, Hou N, Cheng X, Schatten H, Xu X, Yang X, Sun QY. 2015. Loss of protein phosphatase 6 in oocytes causes failure of meiosis II exit and impaired female fertility. $J$ Cell Sci, 128:3769-3780.

Huan Y, Xie B, Liu S, Kong Q, Liu Z. 2015. A novel role for DNA methyltransferase 1 in regulating oocyte cytoplasmic maturation in pigs. PloS one, 10:e0127512. Iwamoto M, Onishi A, Fuchimoto D, Somfai T, Takeda K, Tagami T, Hanada H, Noguchi J, Kaneko H, Nagai T, Kikuchi K.. 2005. Low oxygen tension during in vitro maturation of porcine follicular oocytes improves parthenogenetic activation and subsequent development to the blastocyst stage. Theriogenology, 63:1277-1289.

Kang JT, Atikuzzaman M, Kwon DK, Park SJ, Kim SJ, Moon JH, Koo OJ, Jang G, Lee BC. 2012. Developmental competence of porcine oocytes after in vitro maturation and in vitro culture under different oxygen concentrations. Zygote, 20:1-8.

Kong Q, Xie B, Li J, Huan Y, Huang T, Wei R, Lv J, Liu S, Liu Z. 2014. Identification and characterization of an oocyte factor required for porcine nuclear reprogramming. J Biol Chem, 289:6960-6968.

Krisher RL. 2004. The effect of oocyte quality on development. J Anim Sci, 82(E-Suppl):E14-23.

Li HK, Kuo TY, Yang HS, Chen LR, Li SS, Huang HW. 2008. Differential gene expression of bone morphogenetic protein 15 and growth differentiation factor 9 during in vitro maturation of porcine oocytes and early embryos. Anim Reprod Sci, 103:312-322.

Lonergan P, Rizos D, Gutierrez-Adan A, Fair T, Boland MP. 2003. Oocyte and embryo quality: effect of origin, culture conditions and gene expression patterns. Reprod Domest Anim, 38:259-267.

MacLennan M, Crichton JH, Playfoot CJ, Adams IR. 2015. Oocyte development, meiosis and aneuploidy. Semin Cell Dev Biol, 45: 68-76.

McNatty KP, Smith P, Moore LG, Reader K, Lun S, 
Hanrahan JP, Groome NP, Laitinen M, Ritvos O, Juengel JL. 2005. Oocyte-expressed genes affecting ovulation rate. Mol Cell Endocrinol, 234:57-66.

Miyamoto K, Nagai K, Kitamura N, Nishikawa T, Ikegami H, Binh NT, Tsukamoto S, Matsumoto M, Tsukiyama $T$, Minami $N$, Yamada $M$, Ariga $H$, Miyake M, Kawarasaki T, Matsumoto K, Imai $\mathbf{H}$. 2011. Identification and characterization of an oocyte factor required for development of porcine nuclear transfer embryos. Proc Natl Acad Sci U S A, 108:70407045.

Park JI, Hong JY, Yong HY, Hwang WS, Lim JM, Lee ES. 2005. High oxygen tension during in vitro oocyte maturation improves in vitro development of porcine oocytes after fertilization. Anim Reprod Sci, 87:133-141.

Pocar P, Brevini TA, Perazzoli F, Cillo F, Modina S, Gandolfi F. 2001. Cellular and molecular mechanisms mediating the effects of polychlorinated biphenyls on oocyte developmental competence in cattle. Mol Reprod Dev, 60:535-541.

Roberts R, Franks S, Hardy K. 2002. Culture environment modulates maturation and metabolism of human oocytes. Hum Reprod, 17:2950-2956.

Sha W, Xu BZ, Li M, Liu D, Feng HL, Sun QY. 2010 Effect of gonadotropins on oocyte maturation in vitro: an animal model. Fertil Steril, 93:1650-1661.

Sun QY, Nagai T. 2003. Molecular mechanisms underlying pig oocyte maturation and fertilization. $J$ Reprod Dev, 49:347-359.

Sun QY, Wu GM, Lai L, Park KW, Cabot R, Cheong HT, Day BN, Prather RS, Schatten H. 2001. Translocation of active mitochondria during pig oocyte maturation, fertilization and early embryo development in vitro. Reproduction, 122:155-163.

Swain JE. 2010. Optimizing the culture environment in the IVF laboratory: impact of $\mathrm{pH}$ and buffer capacity on gamete and embryo quality. Reprod Biomed Online, 21:6-16.

Tarkowski AK, Wroblewska J. 1967. Development of blastomeres of mouse eggs isolated at the 4- and 8-cell stage. J Embryol Exp Morphol, 18:155-180.

Torner H, Brussow KP, Alm H, Ratky J, Pohland R, Tuchscherer A, Kanitz W. 2004. Mitochondrial aggregation patterns and activity in porcine oocytes and apoptosis in surrounding cumulus cells depends on the stage of pre-ovulatory maturation. Theriogenology, 61:1675-1689.

Vajta G, Holm P, Greve T, Callesen H. 1997. The submarine incubation system, a new tool for in vitro embryo culture: a technique report. Theriogenology, 48:1379-1385.

Van Blerkom J, Runner MN. 1984. Mitochondrial reorganization during resumption of arrested meiosis in the mouse oocyte. Am J Anat, 171:335-355.

Wang Q, Sun QY. 2007. Evaluation of oocyte quality: morphological, cellular and molecular predictors. Reprod Ferti Dev, 19:1-12.

Watson AJ. 2007. Oocyte cytoplasmic maturation: a key mediator of oocyte and embryo developmental competence. J Anim Sci, 85:E1-3.

Wessel GM, Conner SD, Berg L. 2002. Cortical granule translocation is microfilament mediated and linked to meiotic maturation in the sea urchin oocyte. Development, 129:4315-4325.

Wrenzycki C, Stinshoff H. 2013. Maturation environment and impact on subsequent developmental competence of bovine oocytes. Reprod Domest Anim, 48(Suppl 1):38-43.

Yan C, Wang P, DeMayo J, DeMayo FJ, Elvin JA, Carino C, Prasad SV, Skinner SS, Dunbar BS, Dube JL, Celeste AJ, Matzuk MM. 2001. Synergistic roles of bone morphogenetic protein 15 and growth differentiation factor 9 in ovarian function. Mol Endocrinol, 15:854-866.

Yang CR, Miao DQ, Zhang QH, Guo L, Tong JS, Wei Y, Huang X, Hou Y, Schatten H, Liu Z, Sun QY. 2010. Short-term preservation of porcine oocytes in ambient temperature: novel approaches. PloS one, 5:e14242.

Zhang X, Miao Y, Zhao JG, Spate L, Bennett MW, Murphy CN, Schatten H, Prather RS. 2010. Porcine oocytes denuded before maturation can develop to the blastocyst stage if provided a cumulous cell-derived coculture system. J Anim Sci, 88:2604-2610.

Zheng Z, Zhao MH, Jia JL, Heo YT, Cui XS, Oh JS, Kim NH. 2013. Knockdown of maternal homeobox transcription factor SEBOX gene impaired early embryonic development in porcine parthenotes. $J$ Reprod Dev, 59:557-562. 